\title{
Fountain Codes in Broadcast Channel: Transmission and Joint BP Decoding Algorithm
}

\author{
Kai Fang ${ }^{\mathrm{a}}$, Dazhuan $\mathrm{Xu}^{\mathrm{b}}$ and Shengkai $\mathrm{Xu}$ \\ School of Nanjing University Of Aeronautics And Astronautics, Nanjing 211106, China \\ afranckcool2@163.com, bxudazhuan@nuaa.edu.cn, cxushengkai@nuaa.edu.cn
}

Keywords: fountain codes, broadcast channel, incremental encoding, joint decoding.

\begin{abstract}
In this paper, we study systematic Luby Transform (SLT) codes over broadcast channel with the white Gaussian noise. First, we adopt the incremental encoding and decoding method to transmit information. To reduce the multiple users' interference and improve the throughput performance of the system, we proposed a joint BP algorithm which combines the likelihood ratio update with the BP decoding. In addition, we derive the likelihood ratio iterative updating expression. Simulation results show that the proposed decoding method can provide better throughput performance of SLT codes over broadcast channel.
\end{abstract}

\section{Introduction}

Digital fountain code [1-4] is a new realization of erasure code, which is proposed for large scale network data dissemination and reliable transmission. Different from the traditional erasure codes, digital fountain codes can generate arbitrary number of code-words according to a certain probability distribution, which is not limited by the code rate. The receiver does not need to care about the specific coding sequence and group. So long as it gets enough encoding packets, the receiver can realize successful decoding. Luby et al first proposed the concept of digital fountain [1] in 1998but did not construct a practical digital fountain code. In 2002, Luby further proposed the first practical digital fountain codes, LT codes and designed the practical degree distribution, the robust soliton distribution [2]. LT codes can approach the channel capacity in arbitrary erasure channel, whereas their decoding complexity is nonlinear with respect to the length of source symbols. To provide better performance for fountain codes, Shokrollahi proposed Raptor codes [3] with linear decoding complexity.

Building on top of the coding scheme over the BEC [1, 4], the idea of fountain codes can also be generalized to other channels such as binary symmetric channels (BSC), additive white Gaussian noise (AWGN) channels, and fading channels, which have been shown to achieve good decoding performance [5, 6]. Similar to LDPC codes [7], to recover the input symbols, the belief propagation (BP) decoding algorithm is used on noisy channels [8]. However, LT codes still suffer error floor on noisy channels [9].

Recently, systematic codes have received extensive attention since it is widely accepted as an efficient coding scheme in practical applications. However, LT codes and Raptor codes in prior works $[10,11]$ are not originally designed systematically. As pointed out in [12], the performance of non-systematic LT codes is degraded on noisy channels because of low density of the generator matrix, when using the BP decoding algorithm. Ref. [12-15] proposed various ways to make LT and Raptor codes systematic. Among [12-15] the most straightforward method to construct systematic fountain codes is to transmit input symbols prior to encoding symbols. To improve the bit-error-ratio (BER) performance of LT codes, ref. [12] proposed systematic Luby Transform (SLT) codes that expand the generator matrix of LT codes with an identity matrix.

In the broadcast channel, one transmitter delivers the same or different information to the multiple users. Because the broadcast system is very common in practical applications, the broadcast channel is widely studied. However, few researches has been made to apply fountain codes in broadcast channel. Moreover, to promote the practical application of fountain codes in broadcast channel, it is 
necessary that we focus on studying corresponding encoding and decoding methods and the transmission performance. In this paper, we therefore propose encoding and decoding methods for fountain codes in broadcast channel. In addition, we analyze the transmission performance of system fountain codes in broadcast channel through simulations.

The remainder of the paper is organized as follows. Section II introduces the broadcast channel and system model. In section III, we propose the joint belief propagation decoding algorithm based on likelihood ratio updating rules. Some simulation results are shown in Section IV. Section VII concludes the paper.

\section{System model}

This paper focuses on the broadcast channel, which refers to the link between the base station and multiple users. Considering the broadcast channel the single base station and between $K$ users shown in Fig. 1, the messages of all users are superimposed at the base station and $K$ users receive the superimposed information including others', plus the noise $n_{0}$.

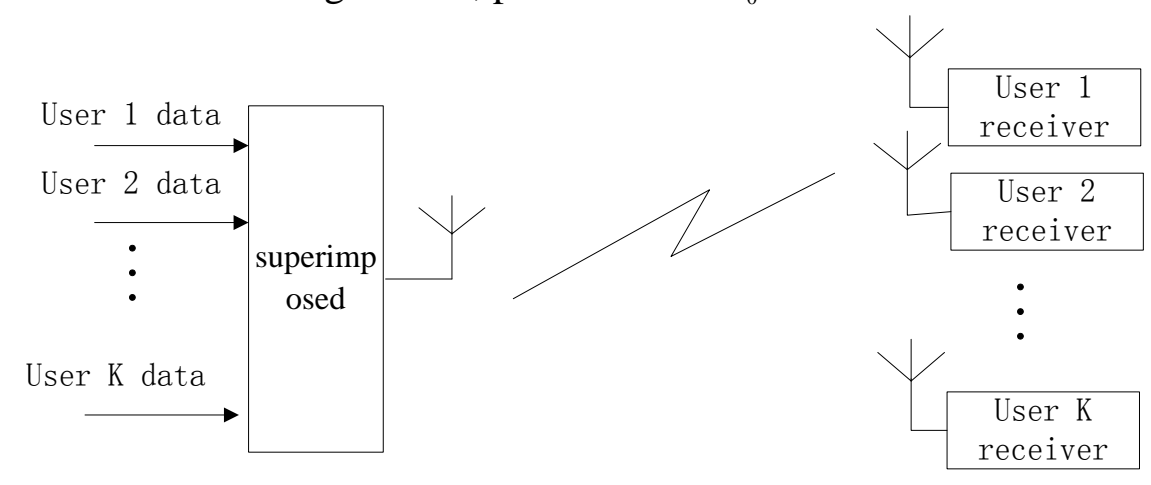

Fig.1 The broadcast channel model.

Assuming that the channel state information is perfectly known at the receiver, i.e., channel estimation is completely correct, the signal received by the user $k$ can be expressed as:

$$
y_{k}=h_{k} \sum_{i=1}^{K} \sqrt{p_{i}} x_{i}+n_{0}
$$

where $h_{k}$ represents the channel impulse response for user $k \cdot p_{i}$ is the transmit power of the user $i$. Let $x_{i}$ denote the transmit signal of user $i$ and $n_{0}$ is the white Gaussian noise.

Based on the characteristics of systematic fountain code, the system adopts the method of incremental encoding and decoding. In order to describe the incremental encoding and decoding process, it is necessary to define the following variables. If the length of the data is $n$ bits and the Incremental overhead of each encoding is $\Delta \varepsilon$, the incremental length per encoding is $n \Delta \varepsilon$. When the users sends the code increment $m$ times and the receiver decodes correctly, the overhead of the system is $m n \Delta \varepsilon$ and the length of encoding is $n(1+m \Delta \varepsilon)$. The incremental encoding and decoding process is as follows:

(1). When overhead is 0 , the user sends data without encoded. If the base station can restore the original data, it will send a successfully-receiving feedback signal to the transmitter. If not, send failed-receiving feedback signal.

(2). When the user receives the failure feedback signal, it sends encoded increment with the length $n \Delta \varepsilon$ which will be cascaded with the previous received signals. And then the cascaded received signals is used to calculate likelihood ratios and decode. If the base station can decode correctly, it will send a success feedback signal to the transmitter. If not, send failed-receiving feedback signal.

(3).If the transmitter receives the failure feedback signal, return step 2 until the base station decode correctly.

System block diagram of fountain codes transmission in multiple access channel is given in figure 2. At the transmitter, $K$ users' information is encoded and modulated. The receiver receives the 
signal from $K$ channels and calculates the input likelihood ratio of $K$ users which will be delivered to the BP decoder. The output likelihood radio of the decoder is used to compute the prior probability of information to update the input likelihood radio of other users. And then the input likelihood radio is sent to the decoder until decoding correctly.

Feedback signal

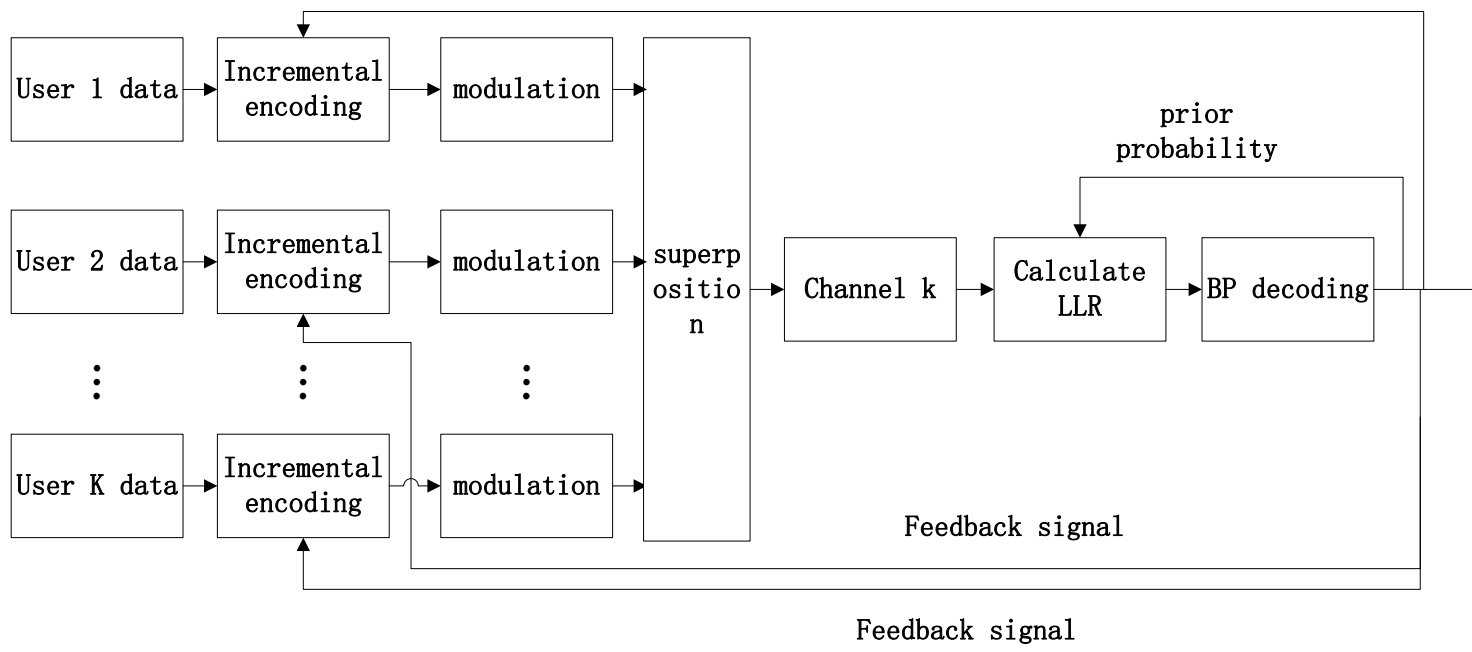

Fig.2 System block diagram of fountain codes transmission in broadcast channel

\section{Joint decoding}

In the wireless channel, the commonly used decoding algorithm of fountain code is BP algorithm. Because the BP decoding algorithm in the logarithmic domain only contains addition and multiplication operations, it is also known as the sum product decoding algorithm (Sum-Product, SP). BP decoding pass the log likelihood ratio information between the input node and the output node to improve the reliability of the decision [16]. The log likelihood ratio of the channel can be defined as [17]:

$$
L L R_{i}=\ln \frac{p\left(\mathrm{x}_{i}=0 \mid \mathrm{y}_{k}\right)}{p\left(\mathrm{x}_{i}=1 \mid \mathrm{y}_{k}\right)}
$$

where $\mathrm{x}_{i}$ is bit information of user $i . p\left(\mathrm{x}_{i}=0 \mid \mathrm{y}_{k}\right)$ is the probability of $\mathrm{x}_{i}=0$ under the condition of the received signal $\mathrm{y}_{k} \cdot p\left(\mathrm{x}_{i}=1 \mid \mathrm{y}_{k}\right)$ represents the probability of $\mathrm{x}_{i}=1$ under the condition of the received signal $\mathrm{y}_{k}$.

We can calculate the initial likelihood ratio of each user by (1)(2):

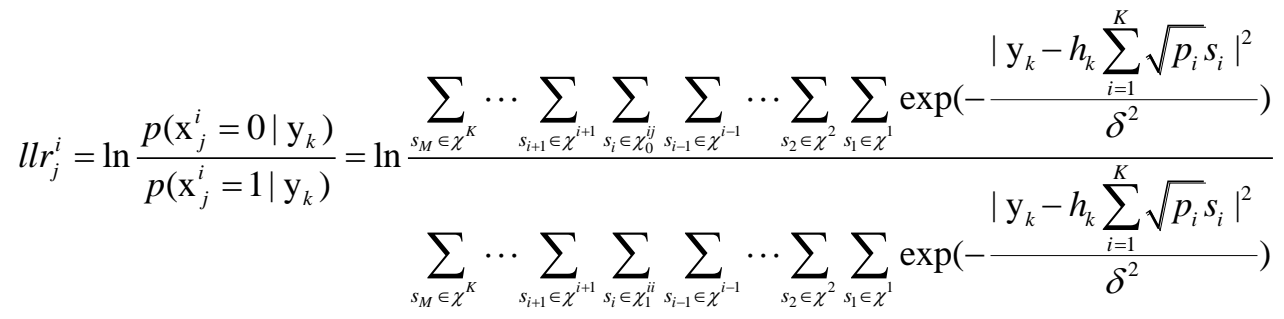

where $l l r_{j}^{i}$ is the likelihood ratio of the bit $j$ of each modulation symbol for the user $i \cdot s_{i}$ represents the modulation constellation point of user $i$. Let $x^{1}, x^{2}, \cdots, x^{i-1}, x^{i+1}, \cdots x^{M}$ denote the collection of all the constellation points for each user except the user $i$ and $\chi_{0}^{i j}$ is a collection of all the constellation points for user $i$ whose bit $j$ is $0 . \mathrm{x}_{j}^{i}$ is the information of bit $j$ for user $i . \delta^{2}$ is the power of the white Gaussian noise.

Note that because the receiver can not get any priori information, the above input likelihood ratio is obtained under the assumption that the probability of the information 0 and 1 is equal. In order to update the input likelihood ratio, this paper calculates the external information and estimates the prior 
probability by the output likelihood ratio of the decoder. this operation will improve the decoding accuracy[18]. The output external information of the decoder is defined as:

$$
v l l r_{j}^{i}=L L R_{j}^{i}-l l r_{j}^{i}
$$

where $l l r_{j}^{i}$ is the input likelihood ratio and $L L R_{j}^{i}$ is the output likelihood ratio. Further, we can get the priori probability.

$$
\left\{\begin{array}{l}
p\left(\mathrm{x}_{j}^{i}=0\right)=\frac{\exp \left(v l l r_{j}^{i}\right)}{1+\exp \left(v l l r_{j}^{i}\right)} \\
p\left(\mathrm{x}_{j}^{i}=1\right)=\frac{1}{1+\exp \left(v l l r_{j}^{i}\right)}
\end{array}\right.
$$

Substituting (5) into (2) to update the input likelihood radio, as follows:

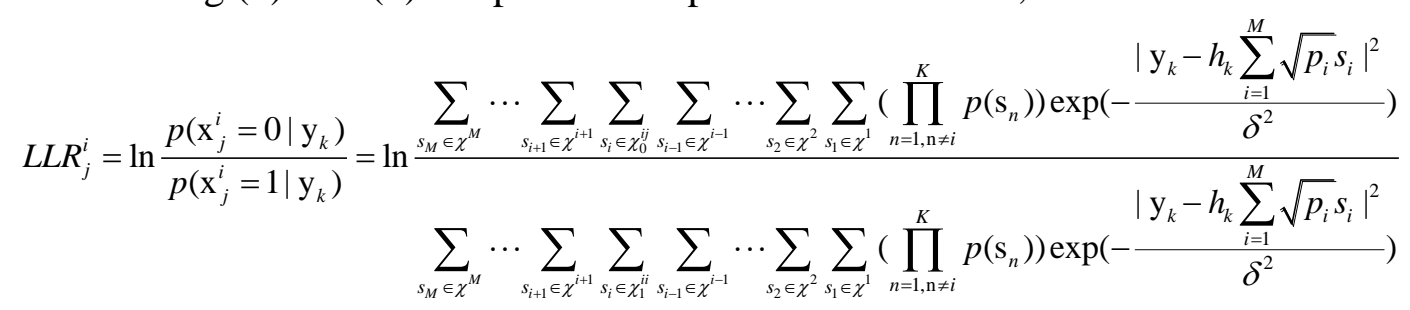

where $p\left(\mathrm{~s}_{n}\right)$ represents the probability that the constellation point $\mathrm{s}_{n}$ of the user $n$ is received.

$$
p\left(\mathrm{~s}_{n}\right)=\prod_{j}^{q} p\left(\mathrm{x}_{j}^{n}\right)
$$

where $p\left(\mathrm{x}_{j}^{n}\right)$ is the probability that the $j$ bit of the constellation point $\mathrm{s}_{n}$ is $\mathrm{x}_{j}^{n} \cdot k$ is the number of bits of information in each constellation point. When the modulation order is $M, q=\log _{2}(M)$.

The likelihood ratio mentioned in (6) will be delivered to the decoder and we repeat the Iterative update decoding process above until decoding correctly or exceeding the maximum number of iterations. After iteration, the likelihood ratio will become more accurate and System throughput will increase.

\section{Numerical results}

In this section, we offer some simulation results of our work. Throughout the whole simulations, we adopt BPSK modulation for SLT codes and the transmit power of two users is set to 1.

We compare the throughput performance of systematic LT code under BP decoding and joint decoding with the signal to noise ratio from $0 \mathrm{~dB}$ to $16 \mathrm{~dB}$, where the degree distribution is

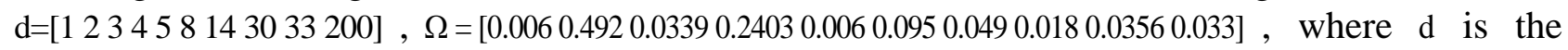
degree and $\Omega$ is the probabilities corresponding to the degree. The channel is Rayleigh channel. The mean results of likelihood ratio are shown in Fig. 3 for the input symbol length 1000 in 1000 simulation runs and the transmit power is $0.5: 0.5$. As we can see, the mean of likelihood ratio for two user be increased largely with the joint decoding algorithm, which cause the higher probability of decoding correct decoding. 

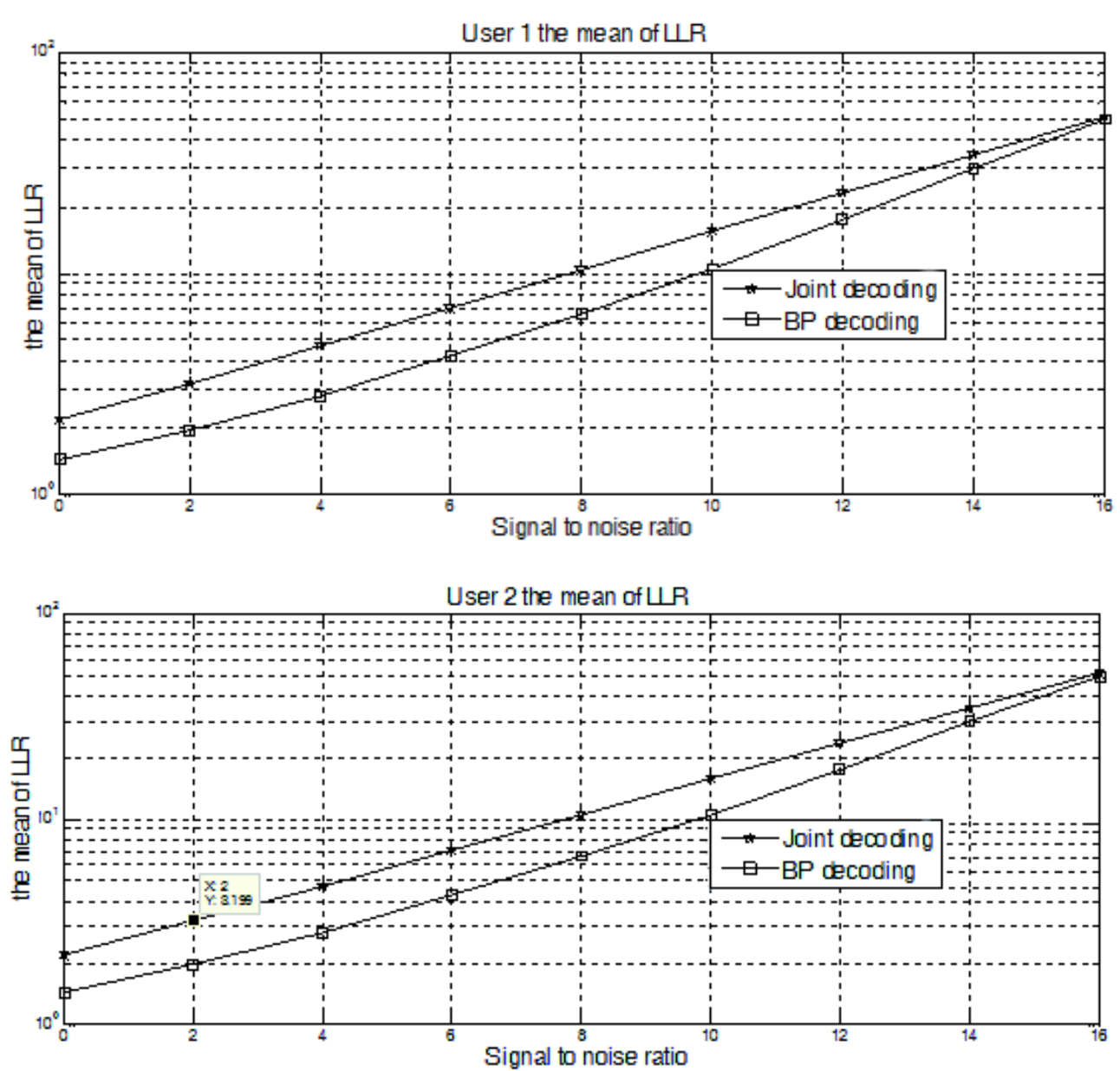

Fig.3 the mean of LLR for two users under joint decoding and BP decoding in Ravleigh broadcast channel

Fig.4 illustrates the throughput performance of the broadcast channel under the joint decoding algorithm and BP decoding algorithm. As we can see, the joint decoding algorithm outperforms the BP decoding algorithm with respect to the total throughput performance in our simulation. In addition, with the increase of signal to noise ratio, the throughput performance of the system is gradually close to the limit of the BPSK modulation.

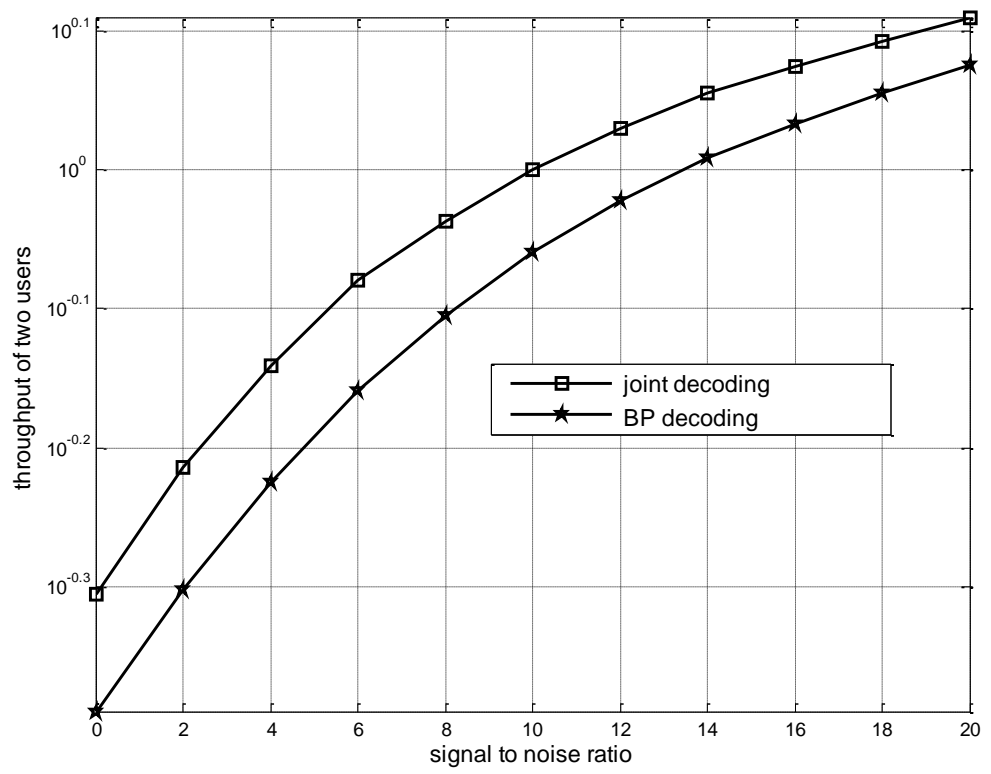

Fig.4 the throughput performance of two users under joint decoding and BP decoding in Rayleigh broadcast channel 


\section{Conclusion}

In this paper, the characteristics of fountain code transmission are studied, and the systematic LT code is applied to the common broadcast channel. We proposed a joint decoding method based on likelihood ratio iterative update and BP algorithm in broadcast channel. The numerical simulation results show that the joint decoding can greatly improve the system throughput performance.

\section{References}

[1] Byers J W, Luby M, Mitzenmacher M, et al. A Digital Fountain Approach to Reliable Distribution of Bulk Data [J]. ACM SIGCOMM Computer Communication Review, 1998, 28(4): 56-67.

[2] Luby M. LT codes [C]. Proc. 2002 IEEE Symp. Foundations of Computer Science (FOCS), Vancouver, Canada, 2002: 271-280.

[3] Shokrollahi A. Raptor codes [J]. IEEE Transactions on Information Theory, 2006, 52(6): 2551-2567.

[4] Mackay D J C. Fountain codes [J]. IEE Proceedings-Communications, 2005, 152(6): 1062-1068.

[5] Palanki R, Yedidia J S. Rateless codes on noisy channels[C]. Information Theory, 2004. ISIT 2004. Proceedings. International Symposium on. IEEE, 2004:37.

[6]Castura J, Mao Y, Draper S. On Rateless Coding over Fading Channels with Delay Constraints[C]. IEEE International Symposium on Information Theory. 2006:1124-1128.

[7]Baldi M. Low-Density Parity-Check Codes[M]. Lowdensity parity-check codes. Мир, 1966:21 28.

[8]Jenkač H, Mayer T, Stockhammer T, et al. Soft decoding of LT-codes for wireless broadcast[C]. Ist Summit. 2005.

[9]Hussain I, Xiao M, Rasmussen L K. Error Floor Analysis of LT Codes over the Additive White Gaussian Noise Channel[C]. IEEE Global Telecommunications Conference. IEEE, 2011:1-5.

[10]Luby M. LT Codes[C]. Symposium on Foundations of Computer Science. IEEE Computer Society, 2002:271 - 280.

[11]Shokrollahi A. Raptor Codes[J]. Information Theory IEEE Transactions on, 2009, 52(6):2551 2567.

[12]Nguyen T D, Yang L L, Hanzo L. Systematic Luby Transform Codes and Their Soft Decoding[J]. IEEE Workshop on Signal Processing Systems, 2007:67-72.

[13]Shokrollahi A. Theory and applications of Raptor codes[J]. Mathknow Mathematics Applied Sciences \& Real Life, 2009, 3:59-89.

[14]Luby M G, Shokrollahi M A. Systematic encoding and decoding of chain reaction codes,EP 1552617 A2[P]. 2005.

[15]Yuan X, Ping L. On Systematic LT Codes.[J]. IEEE Communications Letters, 2008, 12(9):681-683.

[16]Zhu Hongjie, Pei Yukui, Lu Jianhua. Algorithm improving the decoding performance of fountain codes [J]. Tsinghua Science and Technology, 2010(4):609-612.

[17] Etesami O, Shokrollahi A. Raptor codes on binary memoryless symmetric channels[J]. IEEE Transactions on Information Theory, 2006, 52(5):2033-2051.

[18] T.J.Richardson and R.L.Urbanke, The capacity of low-density parity-check codes under message-passing decoding. IEEE Trans. Inf. Theory. Vol. 47(2001) NO. 2, p.599-618. 